\title{
Dissection of the Celiac Artery
}

Jason Arthur, MD, MPH; Eric Iheme, MD; Stephen Topp, MD; Petra Duran, MD

\section{A 41-year-old man presented to the ED with a 4-day history of epigastric pain.}

\section{Case}

A 41-year-old man presented to our ED with a 4-day history of epigastric pain radiating to the bilateral flanks and back. His medical history was significant for hyper-

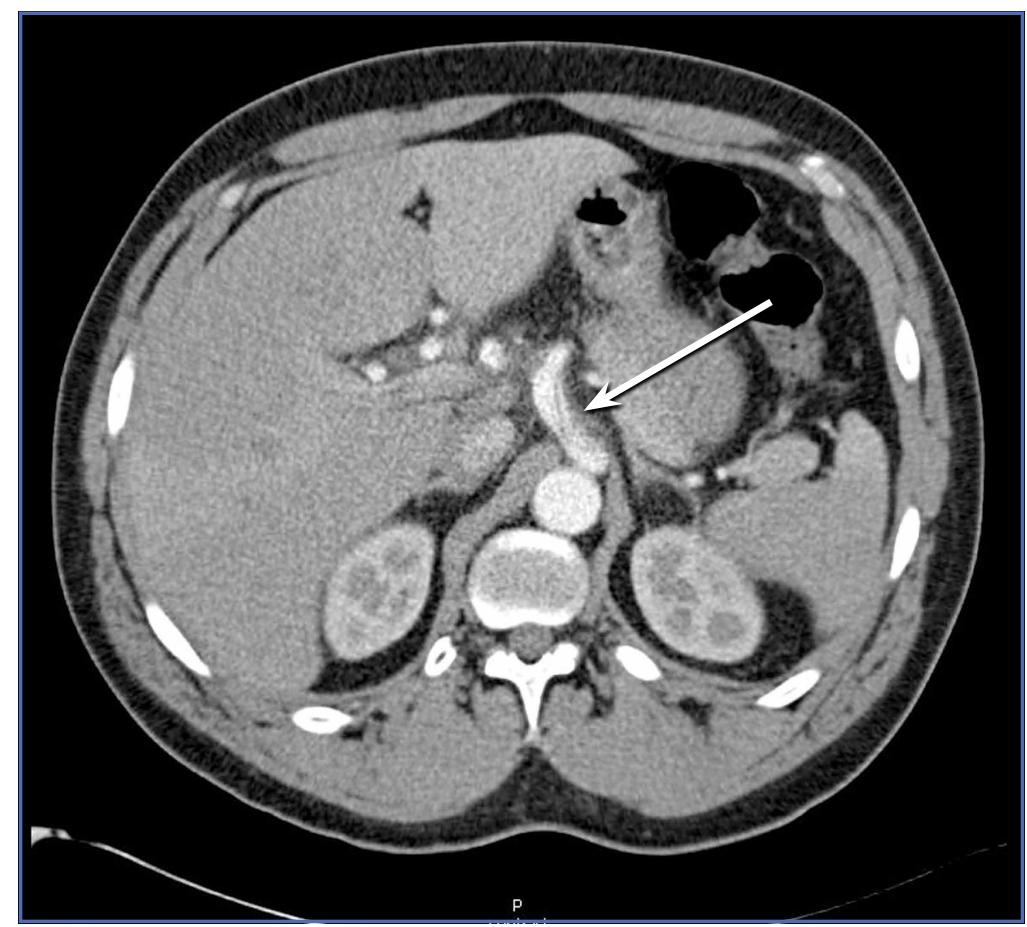

Figure 1. Computed tomography angiography of the patient's abdomen showing the celiac artery with the dissection flap (white arrow). tension, for which he was prescribed isosorbide dinitrite $30 \mathrm{mg}$ four times per day; however, he reported that he did not regularly take this medication.

The patient had visited our ED 3 days earlier with the same complaint. Since his blood pressure (BP) reading at the first ED presentation was 213/141 $\mathrm{mm} \mathrm{Hg}$, he had been admitted for hypertensive urgency. The patient's BP was controlled with antihypertensive agents during his stay, but he continued to experience epigastric pain. A basic work-up for abdominal pain was ordered, the results of which were normal. Based on these findings, the patient's pain was attributed to gastritis, and he was discharged home with instructions to return to the ED if his pain became worse or persisted.

At both ED presentations, the patient denied experiencing any nausea, vomiting, diarrhea, or chest pain. At the second presentation, his triage BP was 158/106 mm Hg. A chest X-ray, complete blood count (CBC), basic metabolic profile (BMP), hepatic panel, and lipase evaluation were all unremarkable, with the exception of a mild increase in creatinine to $1.38 \mathrm{mg} / \mathrm{dL}$. A point-of-care (POC) ultrasound study of the aorta was normal. Given the patient's

Dr Arthur is a postgraduate year 2 resident, department of emergency medicine, University of Florida, College of Medicine-Jacksonville. Dr Iheme is a postgraduate year 3 resident, department of emergency medicine, University of Florida, College of Medicine-Jacksonville. Dr Topp is an assistant professor, department of emergency medicine, University of Florida, College of Medicine-Jacksonville. Dr Duran is an assistant professor, department of emergency medicine, University of Florida, College of Medicine-Jacksonville.

Authors' Disclosure Statement: The authors report no actual or potential conflict of interest in relation to this article.

DOI: 10.12788/emed.2017.0001 


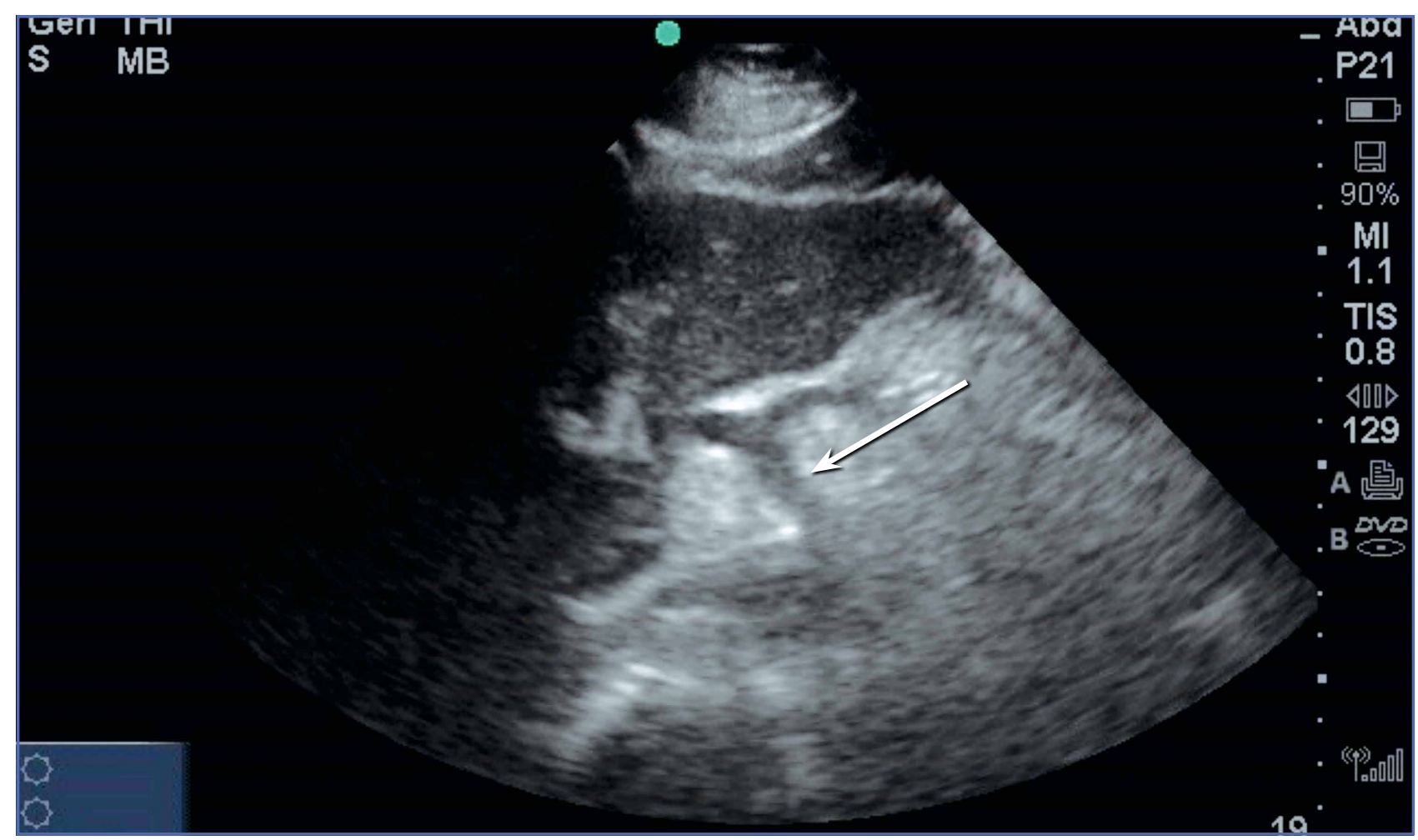

Figure 2. Ultrasound image of the patient's aorta showing the celiac artery but no visualization of the dissection flap (white arrow).

recent hypertensive episode, a computed tomography angiography (CTA) of the chest and abdomen was also obtained, which revealed an isolated $4.5-\mathrm{cm}$ dissection of the celiac artery (DCA) with filling defect in the hepatic artery (Figure 1).

Based on the CTA findings, a nicardipine infusion was immediately started, and the patient was admitted to the medical intensive care unit (MICU). Because his heart rate was in the range of 60 beats/min, an esmolol infusion was not required. Prior to transferring the patient to MICU, a second ultrasound study of the aorta was performed by our fellowship-trained director of emergency medicine ultrasound. Despite the excellent views taken of the aorta, celiac trunk, and hepatic and splenic arteries (Figure 2), the dissection was not visible on either of the two ultrasound images.

In the MICU, the patient's BP was stabilized on hospital day 2, and he was tran- sitioned to oral antihypertensive medications. He was also started on a heparin infusion at the recommendation of vascular surgery services.

A repeat CTA of the abdomen taken on hospital day 3 showed an unchanged dissection in the celiac axis extending into the hepatic artery. The vascular surgeon recommended strict BP control, anticoagulation therapy, and a vascular surgery follow-up with a repeat CTA of the abdomen in 6 months.

On hospital day 6, repeat serial CBC, BMP, and hepatic panels revealed only slight increases in aspartate transaminase to $88 \mathrm{U} / \mathrm{L}$ and alanine aminotransferase to $117 \mathrm{U} / \mathrm{L}$. The patient was transitioned to enoxaparin and discharged home on hospital day 6, and instructed to follow-up with his primary care physician for transition to warfarin. Unfortunately, this patient was lost to follow-up. 


\section{Discussion}

Isolated DCA is a rare cause of abdominal pain. The first documented case of isolated DCA is often incorrectly attributed to Bauersfeld's 1947 case series on dissections, but that report described superior mesenteric artery dissection rather than a celiac artery dissection. Watson's ${ }^{2} 1956$ dissection series is also incorrectly cited as the first DCA, but that series described a dissection of the splenic artery, which is a branch of the celiac artery. In a 1959 series, Foord and Lewis ${ }^{3}$ described what is most likely the first report of DCA as an incidental finding at autopsy. More frequent descriptions in recent years are thought to be due to the routine use of abdominal CTA. ${ }^{4}$

Dissection of the celiac artery is a rare occurrence, with less than 100 cases reported, and little evidence exists to guide its management. ${ }^{5}$ These dissections represent $36.8 \%$ of all visceral artery dissections, ${ }^{6}$ which themselves are less common than renal, carotid, and vertebral artery dissections. ${ }^{7}$ Dissection of visceral arteries occurs predominantly in men and more often in middle-aged patients. ${ }^{8}$ Risk factors for DCA are thought to mirror risk factors for dissection of other arteries, including atherosclerotic disease, hypertension, connective tissue disorders, trauma, vasculitis, and pregnancy. ${ }^{9-11}$

\section{Signs and Symptoms}

Patients with DCA typically present with sudden onset of epigastric, flank, and/or chest pain, though $50 \%$ of patients may be asymptomatic. ${ }^{12}$ This pain is easily overlooked because the physical examination and laboratory studies are typically unremarkable.$^{13}$ Fortunately, DCA is rarely accompanied by fatal organ dysfunction due to collateral flow from other vessels. ${ }^{14}$

\section{Diagnosis and Management}

While CTA with contrast is considered the mainstay of diagnosis of DCA, ${ }^{15}$ optimal treatment for DCA has not been well established. Management options include medical management, operative repair, and endovascular embolization. Medical management is reserved for stable patients without signs of end organ dysfunction. Typical management involves anticoagulation with warfarin for 3 to 6 months and strict BP control accompanied by close surveillance for progression. ${ }^{10,13}$ Some clinicians have argued that anticoagulation therapy may be unnecessary and that risk factor modification and BP control alone may be sufficient. ${ }^{5,6}$ Others have advocated that surgical management should be favored in cases of persistent pain, development of aneurysm, or threatened or compromised flow to end organs. ${ }^{7}$

\section{Point-of-Care Ultrasound}

The American College of Emergency Physicians considers ultrasound of the abdominal aorta a core application of emergency ultrasound. ${ }^{16}$ While sensitivity and specificity of emergency ultrasound for abdominal aortic aneurysm are well established, data supporting its use for screening for dissections are less definitive. With a sensitivity of $67 \%$ to $80 \%$ and a specificity of $99 \%$ to $100 \%$ with visualization of an intimal flap, aortic dissection screening using ultrasound is less reliable than most emergency physicians (EPs) would prefer. $^{17,18}$ There are no published data reporting the sensitivity or specificity of emergency ultrasound for DCA. However, the vascular surgery literature encourages color Doppler ultrasound as part of the initial diagnostic work-up for this rare entity. ${ }^{19}$ While this may seem like an area ripe for emergency ultrasound, it is important to note-as seen in our case-that the site of the dissection is not often seen. Instead, the use of Doppler allows a screening for an abnormal flow pattern suggestive of dissection. ${ }^{20}$

\section{Conclusion}

In our case, both resident EPs and an expert fellowship-trained emergency ultrasound attending physician were unable to 
visualize a dissection-even after knowledge of the lesion was established by CTA. This points out a limitation of emergency ultrasound. While a POC ultrasound may be able to effectively rule in dissections of the aorta and its branches, we cannot reliably rule out these lesions. As EPs continue to expand the use of ultrasound, it is important to balance the desire for efficiency and cost-effectiveness with a high index of suspicion, experience, and clinical acumen.

\section{References}

1. Bauersfeld SR. Dissecting aneurysm of the aorta; a presentation of 15 cases and a review of the recent literature. Ann Intern Med. 1947;26(6):873-889.

2. Watson AJ. Dissecting aneurysm of arteries other than the aorta. J Pathol. 1956;72(2):439-449. doi:10.1002/path.1700720209.

3. Foord AG, Lewis RD. Primary dissecting aneurysms of peripheral and pulmonary arteries: dissecting hemorrhage of media. Arch Pathol. 1959;68:553-577.

4. Neychev V, Krol E, Dietzek A. Unusual presentation and treatment of spontaneous celiac artery dissection. J Vasc Surg. 2013;58(2):491-495. doi:10.1016/ j.jvs.2012.10.136.

5. DiMusto PD, Oberdoerster MM, Criado E. Isolated celiac artery dissection. J Vasc Surg. 2015;61(4): 972-976. doi: 10.1016/j.jvs.2014.10.108.

6. Takayama T, Miyata T, Shirakawa M, Nagawa H. J Vasc Surg. 2008;48(2):329-333. doi:10.1016/j. jvs.2008.03.002.

7. Glehen O, Feugier P, Aleksic Y, Delannoy P, Chevalier JM. Spontaneous dissection of the celiac artery. Ann Vasc Surg. 2001;15(6):687-692.

8. Patel KS, Benshar O, Vrabie R, Patel A, Adler M, Hines G. A major pain in the ... back and epigastrium: an unusual case of spontaneous celiac artery dissection. J Community Hosp Intern Med Perspect. 2014;4(5):23840. doi:10.3402/jchimp.v4.23840.

9. Kang TL, Teich DL, McGillicuddy DC. Isolated, spontaneous superior mesenteric and celiac artery dissection: case report and review of literature. J Emerg Med. 2011;40(2):e21-e25. doi:10.1016/ j.jemermed.2007.12.038.

10. Galastri FL, Cavalcante RN, Motta-Leal-Filho JM, et al. Evaluation and management of symptomatic isolated spontaneous celiac trunk dissection. Vasc Med. 2015;20(4):358-363. doi:10.1177/1358863X15581447.

11. Wang HC, Chen JH, Hsiao CC, Jeng CM, Chen WL. Spontaneous dissection of the celiac artery: a case report and literature review. Am J Emerg Med. 2013;31(6):1000.e3-e5. doi:10.1016/j. ajem.2013.02.007.

12. Oh S, Cho YP, Kim JH, Shin S, Kwon TW, Ko GY Symptomatic spontaneous celiac artery dissection treated by conservative management: serial imaging findings. Abdom Imaging. 2011;36(1):79-82. doi:10.1007/s00261-010-9657-x

13. Wang JL, Hsieh MJ, Lee CH, Chen CC, Hsieh IC. Celiac artery dissection presenting with abdominal and chest pain. Am J Emerg Med. 2010;28(1):111.e3-e5. doi:10.1016/j.ajem.2009.02.023.

14. Takayama Y, Takao M, Inoue T, Yoshimi F, Koyama $\mathrm{K}$, Nagai H. Isolated spontaneous dissection of the celiac artery: report of two cases. Ann Vasc Dis. 2014;7(1):64-67. doi:10.3400/avd.cr.13-00102.

15. Rehman AU, Almanfi A, Nadella S, Sohail U. Isolated spontaneous celiac artery dissection in a 47-year-old man with von Willebrand disease. Tex Heart Inst J. 2014;41(3):344-345. doi:10.14503/THIJ13-3404.

16. American College of Emergency Physicians. Policy statement. Ultrasound Guidelines: Emergency, Point-of-Care, and Clinical Ultrasound Guidelines in Medicine, June 2016. https://www.acep.org/Clinical--Practice-Management/Ultrasound/. Accessed November 15, 2016.

17. Williams J, Heiner JD, Perreault MD, McArthur TJ. Aortic dissection diagnosed by ultrasound. West $J$ Emerg Med. 2010;11(1):98-99.

18. Fojtik JP, Costantino TG, Dean AJ. The diagnosis of aortic dissection by emergency medicine ultrasound. J Emerg Med. 2007;32(2):191-196.

19. Woolard JD, Ammar AD. Spontaneous dissection of the celiac artery: a case report. J Vasc Surg. 2007;45(6):1256-1258.

20. Fenoglio L, Allione A, Scalabrino E, et al. Spontaneous dissection of the celiac artery: a pitfall in the diagnosis of acute abdominal pain. Presentation of two cases. Dig Dis Sci. 2004;49(7-8):1223-1227. 